\title{
Meta
}

Journal des traducteurs

Translators' Journal

Philip C. STINE, ed. (1988): Issues in Bible Translation, UBS

Monograph Series no 3, London/New York/Stuttgart, United

Bible Societies, 296 p.

\section{Guy Couturier}

Volume 35, numéro 2, juin 1990

URI : https://id.erudit.org/iderudit/002435ar

DOI : https://doi.org/10.7202/002435ar

Aller au sommaire du numéro

Éditeur(s)

Les Presses de l'Université de Montréal

ISSN

0026-0452 (imprimé)

1492-1421 (numérique)

Découvrir la revue

Citer ce compte rendu

Couturier, G. (1990). Compte rendu de [Philip C. STINE, ed. (1988): Issues in Bible Translation, UBS Monograph Series no 3, London/New York/Stuttgart,

United Bible Societies, 296 p.] Meta, 35(2), 437-439.

https://doi.org/10.7202/002435ar d'utilisation que vous pouvez consulter en ligne.

https://apropos.erudit.org/fr/usagers/politique-dutilisation/ 
Philip C. STINE, ed. (1988): Issues in Bible Translation, UBS Monograph Series no 3, London/New York/Stuttgart, United Bible Societies, 296 p.

Sous ce titre, Ph. C. Stine publie quinze études qui ont la même intention de discuter de problèmes de traduction de l'Ancien et du Nouveau Testament (hébreu et grec), problèmes tantôt généraux, à cause des langues et des cultures sous-jacentes, tantôt particuliers, à cause surtout de l'ambiguïté ou de l'obscurité des textes en particulier. Leur éditeur a cru bon de les grouper en trois sections différentes, selon l'intention première des différents auteurs. Un premier groupe relève de l'étude d'un genre littéraire particulier, soit le «récit» (discourse), qui est l'objet d'une attention soutenue, surtout depuis la montée des études de type structuraliste. Le deuxième groupe s'adresse aux questions de sociolinguistique, qui a le grand mérite de nous éveiller à cette dimension, aux formes si variées, importante et nécessaire de la société, comme ferment de création et de transformation du langage. Le troisième groupe d'articles se caractérise par une approche commune de différents textes ou groupe de textes dont les difficultés de traduction peuvent être résolues, en partie, par une exégèse de type sociologique, dont la méthode est présentement au cœur même des préoccupations de nombreux exégètes. Chaque article, soigneusement rédigé et édité, est complété par des bibliographies particulières très bien choisies. Nous ne pouvons pas discuter chacune de ces études, ce qui serait dépasser souvent nos compétences, mais il nous semble que certaines d'entres elles méritent une attention réelle, de la part des exégètes autant que des traducteurs.

Une première étude sur le récit pose le problème très général de la traduction ou transposition de ce genre dans le respect des particularités de même genre dans la culture de la langue de traduction (L.M. Zogbo). E.R. Wendland, par contre, s'attarde aux problèmes précis que le traducteur d'un conte particulier, le livre de Ruth, doit comprendre et respecter. L'auteur montre très bien que deux modes distincts de développement sont utilisés par le conteur: il respecte la structure linéaire classique de tout récit (question, explicitation, pointe du récit et résolution), mais il l'échafaude d'après une structure concentrique très savante et bien explicite, par des indices littéraires facilement repérables. Cette structure et ces indices devraient être sensibles dans les traductions ellesmêmes, si on veut respecter le génie du conteur. Les exégètes du livre de Ruth trouveront aussi des observations de grande valeur dans cet article de Wendland. L'étude de D.J. Clark sur Zacharie 9-14 reprend en grande partie l'ouvrage fondamental de P. Lamarche (1961), en précisant quelques points sur la forme littéraire et le milieu social. On s'attardera davantage aux remarques importantes de W.D. Reyburn sur la pratique sémitique caractérisée par l'emploi massif du parallélisme. L'auteur discute brièvement avec pertinence les grands travaux de Kugel, Berlin et Alter, qui restent toujours des ouvrages de référence à ce propos. Il fait de bonnes observations sur les règles à suivre pour que cette loi fondamentale de la poétique hébraïque soit aussi respectée dans les traductions. L'article de E.R. Hope aborde le problème de l'accès aux intentions de l'auteur d'un récit, en particulier celui de Jonas. Les exégètes y trouveront peut-être matière à réflexion, bien que la discussion soit très superficielle; les traducteurs ne seront guère plus éclairés sur les particularités de leur propre travail. Enfin un dernier article (P.A. Noss) s'attarde aux problèmes auxquels doivent faire face certains traducteurs de la Bible en quelques langues africaines quand ils doivent rendre des citations directes ou indirectes; l'intérêt d'une telle étude ne fait pas défaut, bien que sa portée soit plutôt limitée.

Trois études portent sur les implications de la sociolinguistique dans le travail du traducteur de l'Ancien comme du Nouveau Testament. Ph.C. Stine présente une synthèse des travaux récents sur le sujet, en attirant l'attention sur deux dimensions majeures de l'influence de la société sur le langage. D'abord il faut distinguer les différents niveaux 
qui constituent toute société, depuis le niveau populaire jusqu'à l'élite académique. La langue varie d'après ces niveaux, d'où l'importance de cerner celui qui a vu naître tel écrit que nous devons traduire, si nous voulons le respecter dans toutes ses nuances de sens, tout en utilisant un niveau semblable de la société du traducteur. La deuxième dimension relève du style d'un écrit à traduire en regard du type de société qui l'a produit: s'agit-t-il d'une société de culture littéraire ou de culture orale? L'une et l'autre cultures ont leurs caractéristiques propres, que nous n'avons pas encore parfaitement définies, mais dont nous devons déjà tenir compte dans une traduction. Pour Stine une très grande partie de la Bible reflète une société de tradition et de culture orales. Je ne suis pas convaincu qu'il ait raison : les découvertes archéologiques des dernières années nous montrent très clairement qu'Israël a commencé à écrire ses traditions dès les débuts de la monarchie ( $2^{\mathrm{e}}$ moitié du XI $\mathrm{XI}^{\mathrm{e}}$ siècle a.c.). Les deux autres articles offrent un intérêt plus limité, que le présent lecteur ne saurait apprécier à leur juste valeur: H.A. Hatton discute des particularités de la langue thaï dans les formules d'adresse des différents rapports de la société thaïlandaise, alors que G. Ansre pose le problème des influences dialectales sur une des langues de la Rhodésie (Zimbabwe).

Les six dernières études ont été groupées sous le titre général d' '《approches sociologiques» de l'exégèse, méthode relativement nouvelle qui prend de plus en plus d'ampleur. Ces travaux intéresseront surtout les exégètes, bien que les traducteurs pourront en tirer un certain profit. H.P. Scanlin aborde le problème très épineux de l'accès au texte originel (Urtext) de la Bible. Les découvertes de nouveaux manuscrits, surtout ceux de Qumrân (Mer Morte), et une meilleure connaissance des textes sous-jacents aux anciennes traductions (Vorlage) nous obligent à reconnaître que le texte originel n'a cessé de croître, de s'ajuster, d'évoluer jusqu'à son insertion définitive dans un canon, qu'il soit juif ou chrétien. Le concept d'auteur, dans l'Antiquité, n'excluait pas la participation du lecteur, ici toute la communauté des croyants, dans l'élaboration d'une œuvre littéraire. Nous devons sans aucun doute allier davantage les recherches sur l'origine des textes bibliques et leur rassemblement dans un canon des écritures. Il faut ajouter, à notre avis, que de telles découvertes d'ordre strictement textuel et historique ont des implications directes sur notre concept d'inspiration et de révélation; la communauté (société) a peutêtre plus d'importance, à ce niveau, que l'individu qui parle ou écrit. R.A. Bascom pose le problème très général de l'existence de commentaires (midrash) à l'intérieur même d'un texte, sans rien ajouter à ce que nous savions déjà. Par contre on lira avec passion la longue discussion de N.D. Osborn sur un des textes les plus compliqués de toute la Bible, soit le récit de la circoncision de Moïse ou d'un ou deux de ses fils (Exode 4, 2426). Tous les commentateurs du livre de l'Exode ont peiné sur ce texte, de même que tous ses traducteurs depuis la Septante (III ${ }^{\mathrm{e}}$ s. a.c.). La façon dont le texte est structuré et le jeu des pronoms personnels défient tout lecteur de bien distribuer aux bons acteurs les différents détails de ce court récit. Osborn arrive à poser au moins quatorze questions suite à l'ambiguïté générale du texte! Il a sans doute raison de croire que cette ambiguîté a été intentionnelle, dès la première rédaction de cet événement de la vie de Moïse. S'il en est ainsi, est-il juste alors pour un traducteur de lever ces différentes ambiguïtés en identifiant de façon précise les personnages laissés dans le clair-obscur dans le texte hébreu? Si le texte veut laisser planer un doute sur l'incirconcision du fondateur du peuple de Dieu au moment de l'Exode, ne devons-nous pas respecter ce doute dans nos propres traductions? Voilà autant de questions dont nous devrions tenir compte, tant dans nos travaux d'exégèse d'Exode 4, 24-26 que dans les traductions que nous proposons. G.S. Ogden fait aussi ressortir la complexité et l'ambigüté des termes de parenté (père, fils, fille, épouse, etc.) dans la critique de Malachie des prêtres de Jérusalem (Malachie 2, 10-16). Il montre avec beaucoup de vraisemblance que ces difficultés disparaissent si ces termes 
sont compris non comme des références à des rapports de parenté charnelle, mais comme un ensemble de termes symboliques à l'adresse de la «famille ou de la tribu lévitique». Si sa lecture est juste, il faudrait veiller à ce que nos traductions respectent aussi cette portée symbolique par les termes que nous choisirons pour rendre ce texte. R.L. Omanson aborde la question des différences socioculturelles des premières communautés chrétiennes selon qu'elles étaient issues du monde juif ou du monde païen (gréco-romain). R.E. Brown a pu identifier quatre types de réactions de ces deux communautés devant leur obligation ou leur liberté d'observance de certaines pratiques cultuelles juives. L'état actuel de notre connaissance de ces problèmes est suffisamment avancé pour que nous en tenions compte dans notre traduction de tel ou tel terme: il illustre très bien son propos par l'exemple du mot porneia qui peut signifier tantôt «fornication», tantôt «adultère», tantôt «inceste», selon le groupe visé par cet emploi du terme. Enfin $K$. van der Jagt discute avec beaucoup de pertinence le texte difficile de 1 Thimothée 2,15: «la femme est sauvée par sa maternité». L'intelligence comme la traduction de ce court texte sont l'objet de longues discussions depuis longtemps déjà. L'auteur croit qu'il illustre très bien l'importance d'identifier avec précision le milieu visé par cette remarque de Paul ou de l'un de ses disciples. Il croit qu'il s'agit d'un milieu déjà marqué par le courant gnostique, qui range du côté de la chair, donc du mal, toute activité d'ordre sexuel et de procréation; l'auteur veut donc proclamer, en réaction, que la procréation est aussi une œuvre de l'esprit, donc à dimension salvifique. Il faudrait alors tenir compte de cette particularité de doctrine dans nos traductions comme dans nos discussions sur la valeur de la maternité dans l'ensemble de la vie de l'Église.

Il faut être reconnaissant à l'éditeur pour le travail de publication de travaux de grande valeur dans la majorité des cas. Les problèmes soulevés sont importants non seulement pour les traducteurs de la Bible mais aussi pour les exégètes. Il fallait donc que cet ouvrage soit porté à l'attention des uns et des autres.

GUY COUTURIER 